\title{
El aprendizaje activo de la microbiología en el Grado de Podología incrementa el rendimiento académico
}

\author{
Large increases in learning that result with the active teaching methods in \\ Microbiology in Podiatry Degree
}

\author{
María Luisa Gómez-Lus, Carmen Ramos, Pedro Bas, Luis Alou, José Prieto \\ Facultad de Medicina. Area de Microbiología (Departamento de Medicina). Universidad Complutense de Madrid. \\ mlgomezl@ucm.es, carmenramos@ucm.es,pbas@ucm.es, luisalou@ucm.es,jprieto@ucm.es
}

\author{
Correspondencia: \\ María Luisa Gómez-Lus Centelles \\ Area de Microbiología \\ Departamento de Medicina \\ Facultad de Medicina \\ Avda. Complutense s/n \\ E-28040 Madrid \\ Correo electrónico: mlgomezl@ucm.es
}

Fecha de recepción: 25 de diciembre de 2015

Fecha de aceptación: 22 de abril de 2016

Los autores declaran no tener ningún tipo de interés económico o comercial.

\begin{abstract}
RESUMEN
Se ha planteado como objetivo la mejora de la calidad de la docencia de la Microbiología mediante la actualización de la metodología docente, introduciendo como actividad docente el aprendizaje activo basado en preguntas (inquirybased learning:IBL) para conseguir mejorar las competencias que deberán adquirir los estudiantes como parte de su formación integral. En este estudio han participado 55 alumnos de Segundo Curso del Grado de Podología (Curso 2014-2015),y se ha calculado el porcentaje de alumnos que participaron en las 3 pruebas (3 IBL), en 2 (2 IBL), en 1 (1 IBL), y los que no participaron en ninguna y posteriormente se relacionó con las calificaciones obtenidas en la asignatura de Microbiología. Se incluyeron las preguntas IBL que se realizaron en clase en el campus virtual de la asignatura, pero sin incluir la corrección de las mismas.

En los alumnos que realizaron alguna prueba IBL se obtuvieron calificaciones mejores en las preguntas diseñadas para analizar la síntesis de conocimientos y el análisis de datos que en aquellos que no habían participado en ninguna. Al finalizar la actividad se realizó un estudio transversal a través de un cuestionario autocumplimentado en el que se valoraba la opinión de los alumnos sobre el aprendizaje activo mediante IBL valorando positivamente esta actividad para medir el aprendizaje y mejorar la preparación del examen. Consideramos que el uso del campus virtual unido a la actualización en la metodología docente puede mejorar el rendimiento académico de los estudiantes de Microbiología. Palabras clave: microbiología; podología; aprendizaje activo; Campus Virtual; rendimiento.
\end{abstract}

\begin{abstract}
Our focus here is to improve teaching methods in University microbiology classes and the development of educational practices that explicit engage students while enhacing how they learn Microbiology. These practices including inquiry-based learning (IBL) in which students are actively engaged in the learning process for improving the skills to be acquired as part of their effective learning. This study included 55 Microbiology students of Second Year Degree in Podiatry (Academic Year 2014-2015), and calculated the percentage of students who used these resources 3 IBL, 2IBL, IIBL, and that did not participate in any and subsequently related to the student performance in Microbiology exam. The IBL questions that were done in class, were included in the virtual campus, but not including the correction.

Students who prepared IBL scored higher en exam questions designed to test synthesis of knowledge and analysis of data. In order to assess the perceived impact of the active learning, the students were asked to fill out a questionnaire evaluating IBL positively considering that this activity can measure learning and improve exam preparation. We
\end{abstract}


believe that the benefits of active learning methods over lecture instruction are consistent and can increases student performance in Microbiology.

Keywords: microbiology; podiatry; active learning; Virtual Campus; performance.

Referencia normalizada: Gómez-Lus ML, Ramos C, Bas P, Alou L, Prieto J. El aprendizaje activo de la microbiología en el Grado de Podología incrementa el rendimiento académico. Rev. Int. Cienc. Podol. 2016; 10(2): 85-94.

Sumario: 1. Introducción. 2. Material y métodos.3. Resultados. 4. Discusión. Bibliografía. 


\section{INTRODUCCIÓN}

En la Universidad Complutense de Madrid la asignatura de Microbiología se imparte dentro de la materia Biopatología en segundo curso del Grado de Podología1. Se ha planteado como objetivo la mejora de la calidad de la docencia mediante la renovación de la metodología docente cómo en otras Universidades donde la actualización de los objetivos docentes es constante ${ }^{2,3,4}$.

Los alumnos de Microbiología tienen un tiempo limitado para estudiar conceptos complejos que permitan asentar las bases para el diagnóstico de las enfermedades infecciosas en la Podología. Con el objetivo de crear espacios de aprendizaje reflexivo en materias científicas y establecer conexiones entre el aprendizaje teórico y la aplicación práctica profesional, se plantea como un reto la renovación de la metodología docente.

El aprendizaje basado en preguntas (Inquiry-based learning:IBL) es una metodología centrada en el autoaprendizaje guiado por el profesor, en la reflexión que siguen los alumnos para responder a las preguntas planteadas en la explicación de cada tema . De esta forma, se fomenta un pensamiento crítico donde los alumnos se implican de manera activa en el aprendizaje cooperativo mediante la interrelación con los compañeros y el profesor.

El IBL se plantea como un medio diagnóstico para valorar errores conceptuales antes de comenzar la clase y ser un punto de partida para la adquisición e integración de nuevos conocimientos. Mediante esta metodología los alumnos actúan cómo parte activa del aprendizaje ${ }^{5,6}$. Los objetivos del aprendizaje se han definido siguiendo las directrices de la Sociedad Americana de Microbiología (ASM) en la que plantea objetivos sencillos y complejos según la Taxonomía de Bloom ${ }^{7,8}$.Por otra parte se ha comprobado cómo este tipo de aprendizaje incrementa el rendimiento académico mejorando las calificaciones de la asignatura y la valoración de la asignatura ${ }^{9,10}$. Tambien y cómo ya se ha realizado previamente en los estudiantes de Podología ${ }^{1,11}$, se ha consultado la valoración y las opiniones que tienen los alumnos de segundo de Podología sobre el proceso de enseñanza-aprendizaje de la Microbiología. El objeti- vo de este trabajo es comparar los resultados obtenidos en el curso 2013-2014 con el curso 2014-2015, en el que se ha utilizado además el campus virtual cómo espacio de aprendizaje ${ }^{11}$.

\section{MATERIAL Y MÉTODOS}

El estudio se realizó por 55 alumnos que cursaban Biopatología durante el Curso 2014-2015 y que se imparte en $2^{\circ}$ de Grado de la titulación de Podología de la Universidad Complutense de Madrid.

Al inicio de la exposición de un grupo de 3 temas se realizaba una prueba compuesta por varias preguntas a las que podían contestar con verdadero o falso, al finalizar esa unidad temática, contestaban de nuevo esas preguntas y valoraban los aciertos conseguido antes de la clase y después de la clase. Se realizaron 3 tandas de IBL, el primero correspondió a 15 preguntas de la parte de Microbiología general (IBL-A), La segunda a 15 preguntas la parte de Bacteriología (IBL-B) y la tercera a 15 preguntas la parte de Parasitología (IBL-C).

Se ha calculado el porcentaje de alumnos que participaron en las 3 pruebas (3 IBL), en 2 (2 IBL), en 1 ( 1 IBL, y los que no participaron en ninguna y posteriormente se relacionó con las calificaciones obtenidas en la asignatura de Microbiología.

Por otra parte se incluyeron las preguntas IBL que se realizaron en clase en el campus virtual de la asignatura, pero sin incluir la corrección de las mismas.

Posteriomente se desglosó el contenido del examen, que contaba de 30 preguntas, de las que 20 median conceptos básicos desarrollados en la clase teórica o lección magistral y 10 integraban conceptos más complejos ampliados mediante IBL.

Para comparar el porcentaje de aciertos en la parte explicada mediante IBL en los alumnos que entregaron las tres pruebas (IBL A, B y C), se realizó la media de aciertos preclase, postclase y se comparó con el porcentaje de aciertos en el examen. Se empleó el test exacto de Fisher.

Al finalizar actividad se realizó un estudio transversal a través de un cuestionario autocumplimentado en el que se valoraba la opinión 
de los alumnos sobre el aprendizaje reflexivo mediante IBL recibida, resaltando aspectos para mejorar las competencias y habilidades alcanzadas con esta metodología docente. Esta encuesta fue cumplimentada por 26 alumnos.

La participación en todas las pruebas era voluntaria y no se tenía en cuenta para la calificación final.

\section{RESULTADOS}

Del total de alumnos, $10(18,18 \%)$ alumnos participaron y entregaron corregidos las tres tandas de IBL (3 IBL), 18 (32,72\%) alumnos lo hicieron con dos tandas de IBL (2 IBL) y 11 alumnos $(20 \%)$ entregaron solamente una (1
IBL), mientras que 16 alumnos (29,09\%) no entregaron ningún IBL (Figura 1).

En relación a las calificaciones de la asignatura, de los alumnos que realizaron $3 \mathrm{IBL}$, $3(30 \%)$ obtuvieron sobresaliente, $6(60 \%)$ notable, y $1(10 \%)$ aprobado, destacando que en este grupo no hubo ningún suspenso, Del grupo que realizaron 2 IBL, $5(27,7 \%)$ obtuvieron sobresaliente, $8(44,4 \%)$ notable, y $4(22,2 \%)$ aprobado y suspenso 1 (5,5\%). Del grupo que realizó 1 IBL, $2(18,18 \%)$ obtuvieron sobresaliente, $5(45,45 \%)$ notable, y $3(27,27 \%)$ aprobado y suspenso $1(9,09 \%)$.

Del grupo sin IBL, 2 (12,5\%) obtuvo sobresaliente, 4 (25\%) notable, y 7 (43,75\%) aprobado y suspenso 3 (18,75\%) (Tabla 1 y Figura 2).
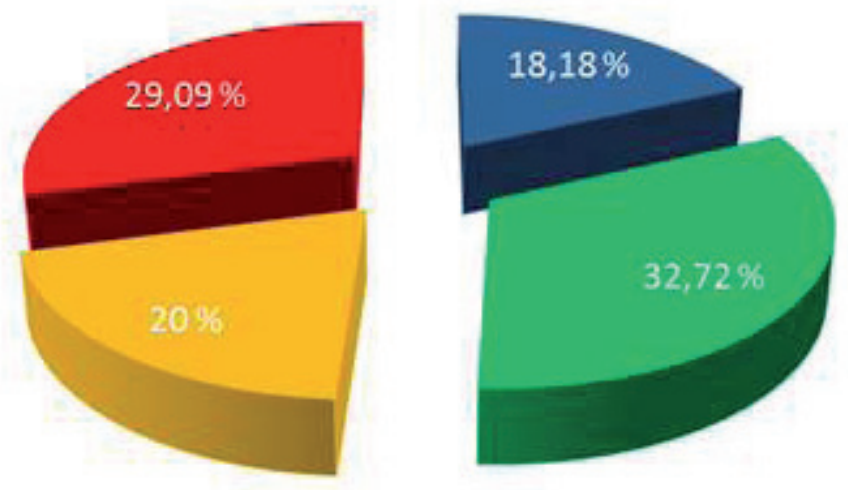

3 IBL

$2 \mathrm{IBL}$

1 IBL

No IBL

Figura 1. Número de alumnos (\%) que realizaron las pruebas IBL.

Tabla 1. Calificaciones agrupadas por la entrega de los diferentes IBL, alumnos que entregaron 3 IBL, 2 IBL , 1 IBL y los que no entregaron ninguno (No IBL).

\begin{tabular}{lccccc} 
& $\begin{array}{c}\text { Total } \\
\mathbf{n}(\%)\end{array}$ & $\begin{array}{c}3 \text { IBL } \\
\text { n (\%) }\end{array}$ & $\begin{array}{c}\text { 2 IBL } \\
\text { n }(\%)\end{array}$ & $\begin{array}{c}\text { 1 IBL } \\
\text { n (\%) }\end{array}$ & $\begin{array}{c}\text { No IBL } \\
\text { n (\%) }\end{array}$ \\
Sobresaliente & $12(21,8)$ & $3(30,0)$ & $5(27,7)$ & $2(18,2)$ & $2(13,4)$ \\
Notable & $23(41,8)$ & $6(60,0)$ & $8(44,4)$ & $5(45,4)$ & $4(26,6)$ \\
Aprobado & $16(20,1)$ & $1(10,0)$ & $4(22,2)$ & $3(27,3)$ & $7(40,0)$ \\
Suspenso & $5(9,1)$ & $0(0,0)$ & $1(5,5)$ & $1(9,1)$ & $3(20,0)$ \\
\hline
\end{tabular}


En relación a los porcentajes de aciertos de las diferentes preguntas IBL que se realizó en el transcurso de las clases, se analizó el incremento del aprendizaje en los alumnos que entregaron las tres pruebas IBL. Se compararon los porcentajes de aciertos al inicio de la clase (pretest) y al finalizar la clase (postest) (Figura 3).

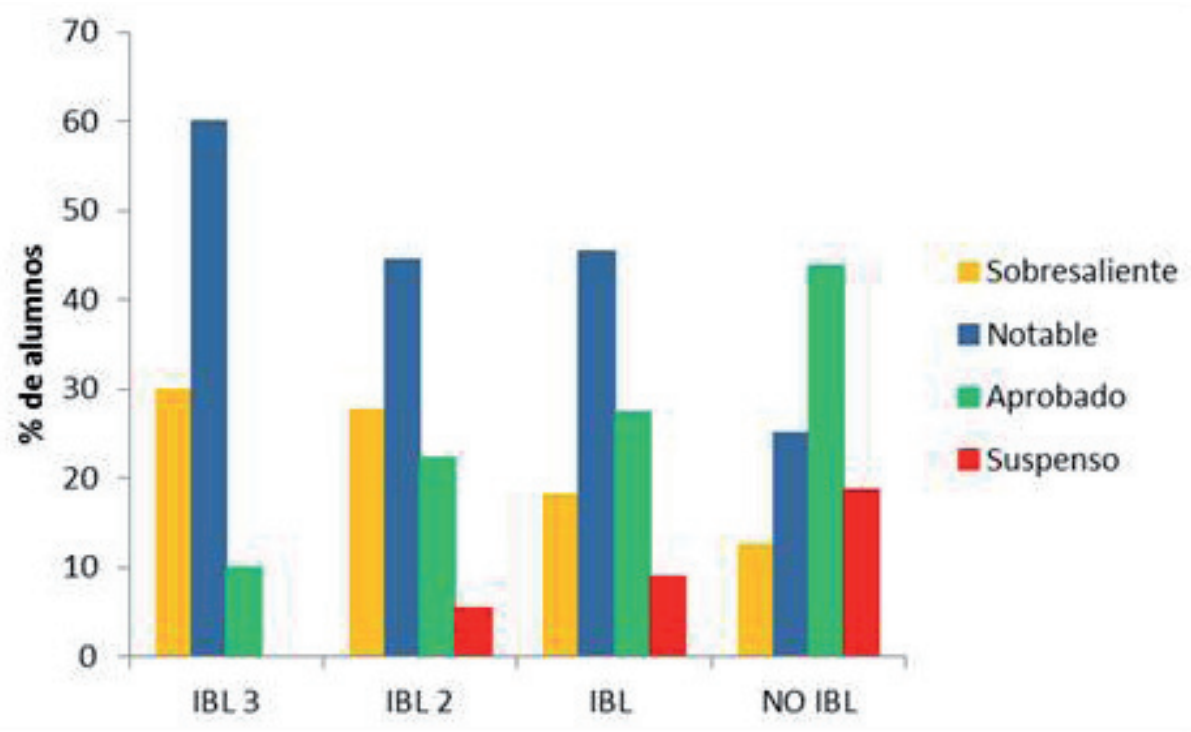

Figura 2. Calificaciones agrupadas por la entrega de los diferentes IBL, alumnos que entregaron 3 IBL, 2 IBL, 1 IBL y los que no entregaron ninguno (no IBL).

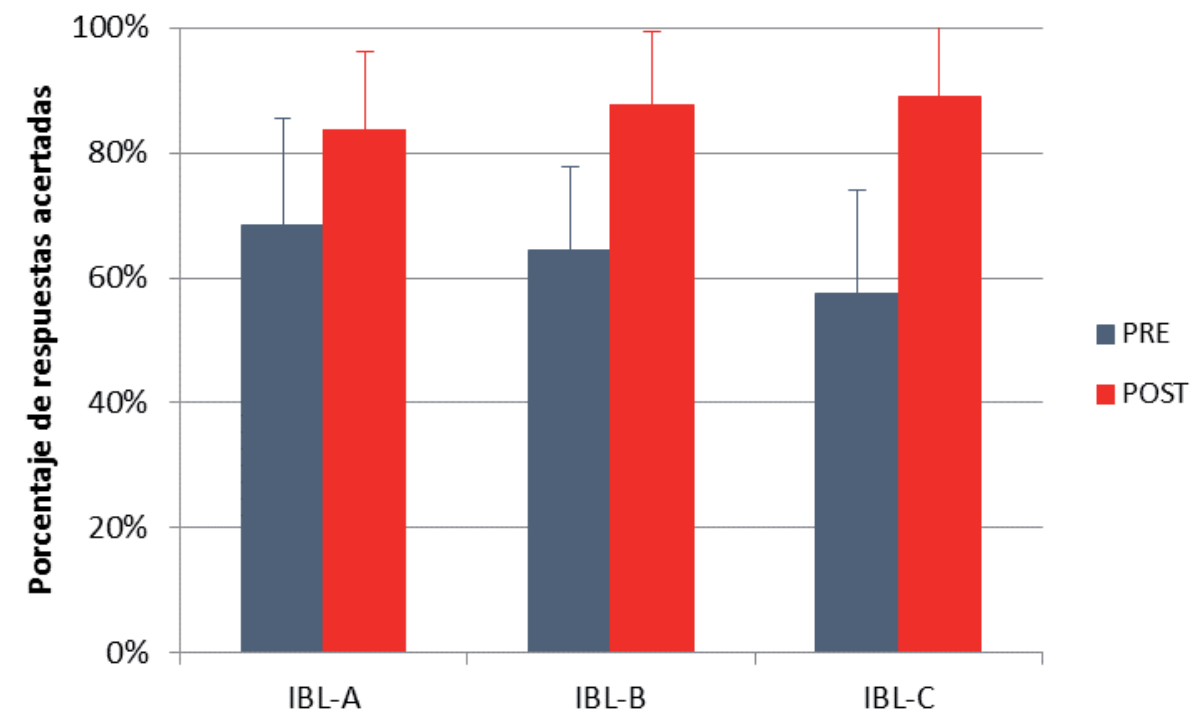

Figura 3. Porcentaje de aciertos antes de la clase (PRE) y despues de la clase (POST) en el primer bloque de IBL (IBL- A), en el segundo bloque de IBL (IBL-B) y en el tercer bloque de IBL (IBL-C). 
En el primer bloque de IBL (IBL A), en el test previo se obtuvo un $68,46 \%$ y en el posterior un 83,66\%; en el segundo bloque de IBL (IBL B) en el test previo se alcanzó un 64,14 $\%$ y en el posterior un $88,06 \%$, mientras que en el tercer bloque de IBL (IBL C) en el test previo se obtuvo un $67 \%$ y en el posterior un $88,93 \%$.Se observaron diferencias significativas $(p<0,0001)$ entre el porcentaje de aciertos obtenido antes de la clase (preclase) y después de la clase (postclase) para todos los IBL (A, B y C).

Dentro del examen de la asignatura, se estructuro en una parte de conceptos más sencillos que explicaron mediante explicación teórica y otra parte de conceptos más complejos que se habían reforzado con el método IBL, al estudiar el índice de aciertos en el examen, comprobando que los aciertos en las preguntas explicadas mediante lección magistral era de un $68,25 \%$ y en grupo explicado con lección magistral mas IBL el porcentaje de aciertos era del $87,45 \%$, cómo pese a ser conceptos mas complicados, los resultados eran mejores (Figura 4). Si comparamos estos resultados con los obtenidos en el curso 2013-2014, el por- centaje de respuestas acertadas en el grupo Teoría + IBL fue significativamente superior $(p<0,0001)$ en el curso 2014-2015 respecto al curso 2013-2014.

Las preguntas del examen correspondientes a los temas estudiados mediante IBL y que en este caso se colgaban en el campus virtual de la asignatura, obtuvieron un porcentaje de aciertos del 87,45 \%.(Figura 5) Se observaron diferencias significativas entre el porcentaje de aciertos obtenido antes de la clase en la media de los tres grupos IBL (preclase - 63,34\%) cuando se comparó con el porcentaje de aciertos después de la clase (postclase - 86,88\%) $(\mathrm{p}<0,0001)$ y el examen de la asignatura (examen-87,45\%) $(\mathrm{p}<0,0001)$. No se observaron diferencias significativas entre el porcentaje después de la clase (postclase) y el examen de la asignatura $(\mathrm{p}=0.25)$.

Al finalizar las clases se valoró el aprendizaje reflexivo basado en preguntas por parte de los alumnos (Tabla 2) en el curso 2014-2015 y comparándolo con el curso 2013-2014, obteniendo la mejor valoración en la pregunta número 8 ya que medían la mejora del aprendizaje en cada una de las unidades temáticas seguida

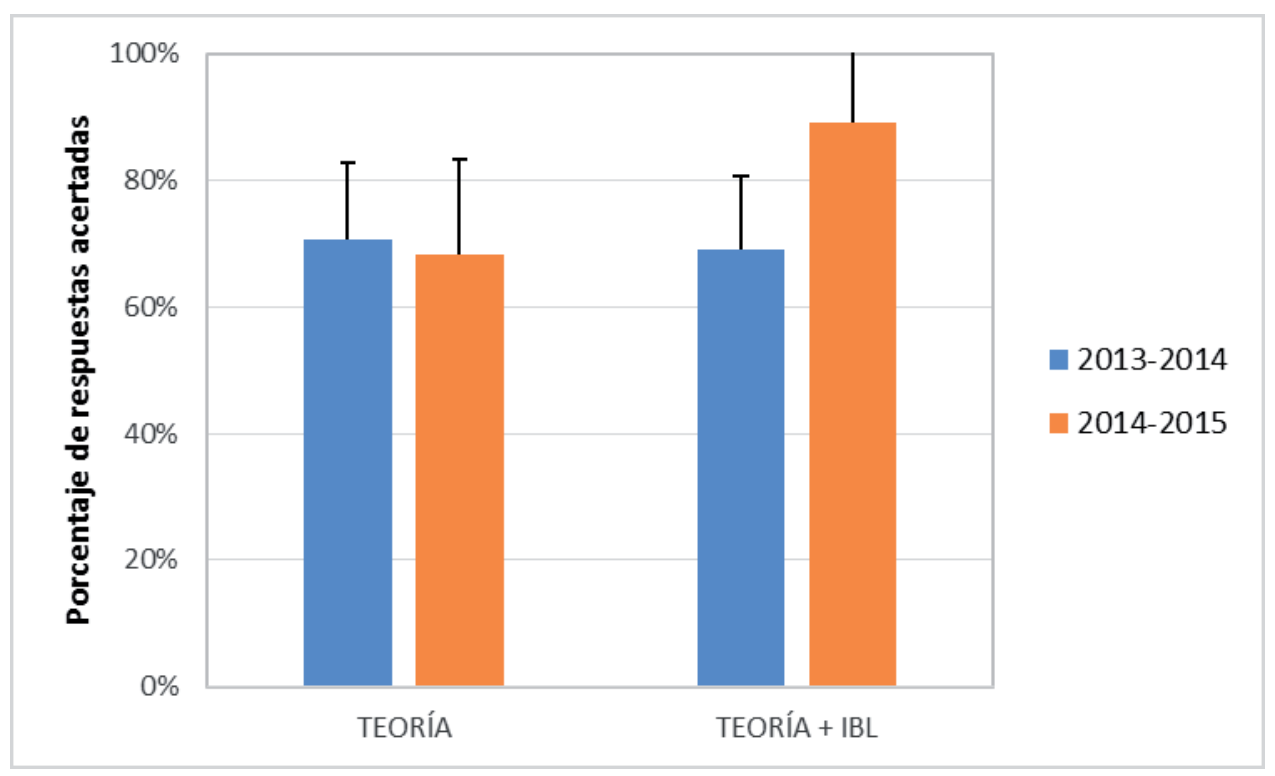

Figura 4. Porcentaje de aciertos en el examen del curso 2013-2014 y 2014-2015 de la asignatura de Microbiología relacionado con el tipo de aprendizaje, teoría de menor complejidad (teoria) o teoria con IBL de mayor compejidad (teoria con IBL). 
de la número 6, ya que esta actividad les había permitido una mejor preparación del examen de la asignatura. Por otra parte en la pregunta numero 7 consideraban que sería útil el incluir este tipo de hojas de trabajo en todos los temas de la asignatura. En relación a los comentarios menos favorables fueron los correspondientes número 4 en el que se valoraba el interés del alumno por la Microbiología, el número 5, en el que consulta si el alumno consideraba que les sería útil para su futura práctica profesional y la número 9 en la que se planteaba si habían detectado errores conceptuales.

En relación a las preguntas abiertas todos los alumnos

informaron que habían mejorado el conocimiento sobre los temas planteados, incrementando su atención en clase y que llevaban al día el estudio y que el aprendizaje activo les permitía autoevaluarse.

\section{DISCUSIÓN}

El porcentaje de alumnos que no entregaron ninguna prueba IBL fue de un $29,09 \%$ des- cendiendo la participación en comparación con los datos obtenidos en el curso 2013-2014 en el cual fue de un $18.36 \%$, posiblemente debido a que al tener los contenidos colgados en el campus virtual, no mantenían en clase la hoja de trabajo con las preguntas. Pese a ello ha incrementado notablemente el interés por parte de los alumnos ya que en trabajos publicados previamente con alumnos de Microbiología General de la Facultad de Medicina de la Universidad Complutense de Madrid el grado de no participación era de un $51 \% \%^{5}$ Al comparar los resultados académicos de los alumnos se observa cómo los alumnos que realizaron voluntariamente las tres pruebas IBL superaron todos la asignatura de Microbiología y el mayor número de suspensos se encontraba en el grupo de los alumnos que nunca habían entregado las hojas de trabajo. En trabajos previos ya demostramos cómo el aprendizaje activo mediante actividades prácticas en la docencia de la Microbiología mejoraron el rendimiento académico de los alumnos, disminuyendo el número de suspensos ${ }^{1}$. En los últimos años se ha comprobado cómo la implementación de métodos de aprendizaje activo han mejorado la

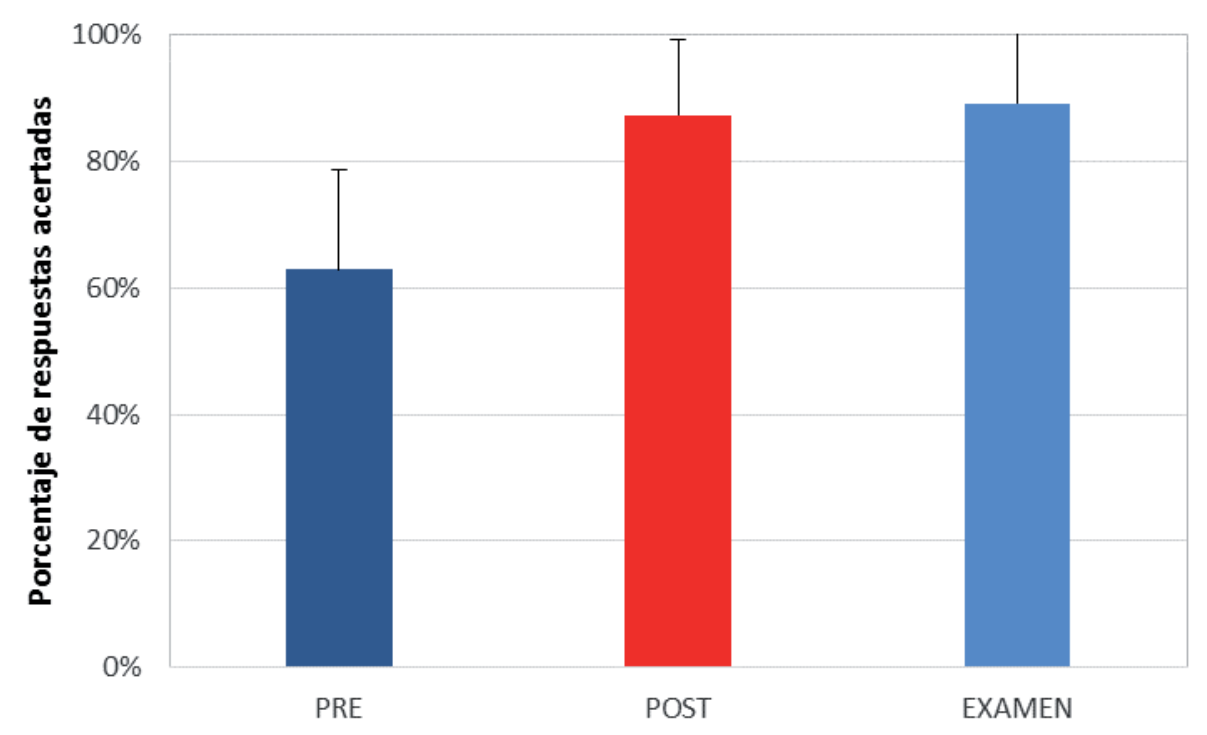

Figura 5. Porcentaje media de respuestas en las preguntas IBL + Teoria acertadas por los alumnos antes (PRE), después de la clase (POST) y en el examen de la asignatura de Microbiología en el curso 2014-2015. 
calidad de la educación obteniendo mejor rendimiento académico que con la lección magistral en la que el alumno escuchaba y tomaba notas mientras que en los métodos de aprendizaje activo el alumno procesa y aplica la información respondiendo preguntas, solucionando y discutiendo problemas y completando hojas de trabajo $^{12,13}$.
Así, la utilización de esta metodología, no sólo es una transferencia de información, sino que puede contribuir a mejorar la calidad de la docencia y considerarse cómo una interacción dentro del proceso de enseñanza y aprendizaje, estableciendo un inventario de conceptos, incentivando el estudio y el aprendizaje cooperativo en la educación científica ${ }^{14}$.

Tabla 2. Valoración de los estudiantes de Podología 2013- 2014 sobre el aprendizaje reflexivo basado en preguntas.

\begin{tabular}{|c|c|c|c|c|c|}
\hline & \multicolumn{5}{|c|}{ n (\%) 2013-2014 / n (\%) 2014-2015 } \\
\hline & 1 & 2 & 3 & 4 & 5 \\
\hline $\begin{array}{l}\text { 1. Ha mejorado mi aprendizaje sobre } \\
\text { el tema }\end{array}$ & & & $4(16) / 3(12)$ & $16(64) / 17(65)$ & $5(20) / 6(23)$ \\
\hline 2. La actividad es clara & & $1(4) / 0(0)$ & $5(20) / 4(15)$ & $9(36) / 13(50)$ & $10(40) / 9(35)$ \\
\hline $\begin{array}{l}\text { 3. He consolidado más mis conoci- } \\
\text { mientos }\end{array}$ & & $1(4) / 0(0)$ & $5(20) / 8(31)$ & $14(56) / 10(38)$ & $5(20) / 8(31)$ \\
\hline $\begin{array}{l}\text { 4. Ha aumentado mi interés por la } \\
\text { Microbiología }\end{array}$ & & $1(4) / 3(12)$ & $7(28) / 10(38)$ & $12(48) / 7(27)$ & $4(16) / 6(23)$ \\
\hline $\begin{array}{l}\text { 5. La actividad me ha permitido re- } \\
\text { flexionar con mis compañeros sobre } \\
\text { algún aspecto útil en mi futura prácti- } \\
\text { ca profesional }\end{array}$ & & $3(12) / 4(15)$ & $11(44) / 7(27)$ & $10(40) / 11(43)$ & $1(4) / 4(15)$ \\
\hline $\begin{array}{l}\text { 6. Esta actividad me ha permitido } \\
\text { prepararme mejor el examen de la } \\
\text { asignatura de Microbiología }\end{array}$ & & & $1(4) / 3(12)$ & $15(60) / 11(43)$ & $9(36) / 12(45)$ \\
\hline $\begin{array}{l}\text { 7. Esta actividad se incluiría en todos } \\
\text { los temas de la asignatura }\end{array}$ & & $0(0) / 1(3)$ & $4(16) / 7(27)$ & $11(44) / 9(35)$ & $10(40) / 9(35)$ \\
\hline $\begin{array}{l}\text { 8. Mediante esta actividad puedo me- } \\
\text { dir mi mejora en el aprendizaje }\end{array}$ & & & $2(12) / 0(\mathbf{0})$ & $12(48) / \mathbf{1 3}(\mathbf{5 0})$ & $11(44) / \mathbf{1 3}(\mathbf{5 0})$ \\
\hline $\begin{array}{l}\text { 9. Esta actividad me ha permitido de- } \\
\text { tectar errores conceptuales }\end{array}$ & & & $4(16) / 4(15)$ & $13(52) / \mathbf{1 3}(\mathbf{5 0})$ & $8(32) / 9(35)$ \\
\hline
\end{tabular}

$1 \quad$ Fuertemente desacuerdo

2 Moderadamente desacuerdo

3 Indiferente

4 Moderadamente de acuerdo

5 Fuertemente de acuerdo 
Al ser una prueba voluntaria, destaca la participación inconstante de los alumnos que no entregan las hojas de trabajo corregidas pero que posiblemente utilicen cómo herramienta de aprendizaje autónomo al no considerar los resultados de esta prueba necesarios para la calificación. Pero debido a los buenos resultados en el examen de las preguntas explicadas mediante IBL que se colgaban en el campus virtual en la mayoría de los alumnos, con un $87,45 \%$ de aciertos, superior a las acertadas en la parte del examen que valoraba los conocimientos adquiridos mediante lección magistral que era de un $68,25 \%$ de aciertos, por lo que las hojas de trabajo IBL que se colgaban en el campus virtual han mejorado considerablemente el porcentaje de aciertos.

La participación en la valoración de este aprendizaje reflexivo en el curso 2014-2015 ha sido de un 47,27 \% (26 alumnos de un total de 55 alumnos), fue algo inferior a la a la obtenida a la del curso 2013-2014 que fue de casi un 51,02\% (25 alumnos de un total de 49 alumnos), mientras que en el trabajo presentado en el Congreso de Docencia y Difusión de la Microbiología 2012 que analizaba la valoración de las clases prácticas en el curso 2011-2012 en los alumnos de Microbiología de Podología fue de un $100 \%$ al ser una prueba obligatoria. Pero estas observaciones, en su mayoría positivas, son interesantes ya que en cada nuevo curso nos planteamos cómo objetivo incrementar los puntos peor valorados, cómo la detección de errores conceptuales y adecuando las preguntas planteadas a su futuro desarrollo profesional cómo Podólogos que permita aumentar su motivación estableciendo una conexión entre la ciencia que se estudia en las aulas y la que se aplica en una clínica podológica.

Consideramos que actualizar la docencia mediante nueva metodología docente permite reforzar el feedback entre profesor y alumno, con la mejora del aprendizaje, tal y cómo se refleja en el presente trabajo con el incremento del rendimiento académico.

\section{BIBLIOGRAFÍA}

1. Gómez-Lus ML, Alou L, Ramos MC, Prieto J, Adaptación al EEES de la enseñanza de la Microbiología en el Grado de Podología, Congreso del grupo de Docencia y Difusión de la Microbiología de la Sociedad Española de Microbiología, Madrid 12 y 13 de Julio de 2012.

2. Johnson MT. Impact of on line learning modules on medical student Microbiology examination scores. 2008. J. Microbiol. Biol. Educ. 2008; 9: 25.29.

3. Lee VS and Hyman M. Helping educational reforms to succeed in a Microbiology Department. Microbe. 2009; 4. 5:219-223.

4. Chaudhry MA. Assessment of Microbiology students' progress with an audience response system, J. Microbiol. Biol. Educ. 2011; 12.2: 200-201.

5. Gómez-Lus ML, Alou L, Ramos MC, Prieto J, Aplicación de nuevas metodologías en el aprendizaje de la Microbiología, XI Congreso Nacional de la SEQ, Madrid, 5-8 Octubre 2011.

6. Rodriguez-Avial C, Gómez-Lus ML, Picazo J. Aplicación del aprendizaje guiado por preguntas a la docencia de Microbiología Médica. Congreso del grupo de Docencia y Difusión de la Microbiología de la Sociedad Española de Microbiología. Madrid 12 y 13 de Julio de 2012.

7. Working Document: ASM GENERAL MICROBIOLOGY LEARNING OUTCOMES. http://www. asm.org/images/Education/Working_Final_ASMGenMicro_LO_-_v_Feb_4_2014.pdf(Último acceso: 22 octubre 2015).

8. ASM Recommended Curriculum Guidelines for Undergraduate Microbiology Education Background http://www.asm.org/images/final_curriculum_guidelines_\%20w._intro_4.12.20-b.pdf (Último acceso: 22 octubre 2015).

9. Peteroy-Kelly MA. A discussion group enhances the conceptual reasoning skills of students enrolled in a large lecture-format introductory biology course. J. Microbiol. Biol. Educ.2007; 8:13-21.

10. Peteroy-Kelly MA. Online pre-laboratory modules enhance introductory biology students' preparedness and performance in the laboratory. J. Microbiol. Biol. Educ. 2010 ;11: 5-13. 
11. Gómez-Lus ML, Ramos C, Alou L, Prieto J. Impacto del aprendizaje de la Microbiología en el Grado de Podología. Rev. Int. Cienc. Podol. 2015; 9 (1), 17-23.

12. Freeman $\mathrm{S}$ et al. Active learning increases student performance in science, engineering, and mathematics. Proc. Nat. Acad. Sci. USA 2014; 111: 8410-8415.

13. Wieman C E. Large-scale comparison of science teaching methods sends clear message. Proc. Nat. Acad. Sci USA 2014; 111: 8319-8320.

14. Wieman C, Gilbert S. Taking a scientific approach to science education, Part II-Changing teaching. Microbe 2015; 10, (15):203-207. 